\title{
Efficiency of Some Chemical and Bio-Insecticides Against Onion Thrips, Thrips Tabaci Lindeman (Thysanoptera: Thripidae)
}

\author{
Alaa M. H. Khozimy ${ }^{1}$, Mohammed A. F. Abuzeid ${ }^{1}$ and Adnan A. E. Darwish ${ }^{1}$
}

\begin{abstract}
Onion thrips, Thrips tabaci (Thysanoptera: Thripidae) is one of the main problems and yields limiting factors in the onion fields (Allium cepa $\mathbf{L}$.). In the present work, an effort was made to study the relative susceptibility of different onion cultivars to infestation with the onion thrips, evaluation of some chemical and bio-insecticides against $T$. tabaci and to study the effect of meteorological factors on its population density. The results showed that T. tabaci infest all the tested cultivars more or less, however cultivar Red Giza showed to be the most tolerant against $T$. tabaci infestation among the other cultivars. Giza 6 was the most susceptible among the cultivars followed by Beheri, Giza 20 and finally White Giza. However, there were no significant different among the White Giza and Red Giza cultivars. The tested insecticides can be arranged according to their efficacy against nymphs of $T$. tabaci in descending order as follows; spinetoram, pyridalyl, imidacloprid, thiamethoxam, lambda-cyhalothrin, dinotefuran, pyriproxyfen, malathion, azadirachtin and finally Beauvaria bassiana. On the other hand, these insecticides can be arranged according to the general means of reduction percentages of T. tabaci adults in a descending order as follows; pyridalyl, spinetoram, thiamethoxam, imidacloprid, lambdacyhalothrin, dinotefuran, pyriproxyfen, malathion, Beauvaria bassiana and azadirachtin. The results of the population trends of $T$. tabaci revealed that the onion thrips had two generations during the onion growing period. There was a significantly negative correlation $(\mathrm{P}<0.05)$ between population density of onion thrips and relative humidity. The results also revealed that the temperature positively and significantly affect $T$. tabaci population in both seasons 2020 and 2021.
\end{abstract}

Key words: Thrips tabaci, Allium cepa, insecticides, population dynamic, onion cultivars.

\section{INTRODUCTION}

Throughout the world, the onion thrips, Thrips tabaci L. (Thysanoptera: Thripidae), is one of the most destructive insect pests to a wide variety of crops (Ananthakrishnan 1973; Afaf, El- Roby, 2018). It is consider a key pest in the onion (Allium cepa) producing areas worldwide (Diaz-Montano, et al., 2011; Gill, et al. 2015; Darwish, 2015). Severe infestation with the adults and nymphs of $T$. tabaci kill onion seedlings. While in the old plants, the severe infestation may cause onion crops to mature early, leading to smaller bulb sizes and subsequently reduce yields (Mahmoud 2008; Boateng, et al., 2014 and Darwish, 2015). The severity of this insect pest can be attributed to its nature as a polyphagous insect pest, large number of annual generations, high reproductive rate, the ability to produce offspring from unfertilized eggs (parthenogenetic reproduction), ability to transmit numerous plant-infecting pathogens as vectors, and development of resistance to chemical insecticides (Morse and Hoddle 2006, Diaz-Montano et al. 2011). Use of resistant or less favorable plant cultivars is considered one of the major components of integrated pest management for an ecological and economic pest control program. Therefore, it is very important to select resistant or at least non preferred onion cultivars at the beginning of the onion thrips integrated pest management program. In onion fields, due to the irruptive outbreaks of onion thrips, the synthetic insecticides have been the most important tools employed for the management of this pest (Morse and Hoddle 2006; Nault and Hessney, 2010; Shah, 2015). Many authors such as, Omar and El-Kholy 2001; Saleh 2004; Sabra et al. 2005; Amro et al. 2009 and Mahmoud, 2011 studied the efficiency of different insecticides on onion thrips in different plant species. The information about either the interaction between onion thrips and resistant onion cultivars or the chemical control of onion thrips is very important for any integrated pest management program. The objective of this work was to study the relative susceptibility of different onion cultivars to the infestation with $T$. tabaci, evaluate some insecticides for controlling $T$. tabaci and to study the population dynamic of $T$. tabaci in onion plants.

\section{MATERIAL AND METHODS}

Susceptibility of different experiment onion cultivars:

In the current experiment five main onion (Allium cepa L.) cultivars namely White Giza, Red Giza, Beheri (red), Giza 6 (red) and Giza 20 (yellow) were cultivated in a private farm at Nobaria district, Beheira Governorate, Egypt during onion growing seasons of 2020 and 2021. This experiment was conducted to study the susceptibility of onion cultivars for the onion thrips. Plants were transplanting in December $14^{\text {th }}, 2019$ (2020

DOI: 10.21608/asejaiqjsae.2021.191176

${ }^{1}$ Plant protection department, Faculty of Agriculture, Damanhour University, Egypt

Received July 15, 2021, Accepted, August 18, 2021. 
season) and December $18^{\text {th }} 2020$ (2021 season). The five Onion cultivars were obtained from Agriculture Research Station. The experimental area was about $2000 \mathrm{~m}^{2}$ and divided into 20 plots (four replicates for each cultivar). The replicates (about $100 \mathrm{~m}^{2}$ ) arranged in Completely Randomized Block Design. The sampling (4 plants from each replicate) was started 21 days postplanting and continues up to the harvest. During the growing seasons, except the use of insecticides, all the recommended agriculture practices were performed as usual.

\section{Efficacy of insecticides experiment:}

Field experiments were conducted to evaluate the effect of ten insecticides on the population density of adults and nymphs of onion thrips, T. tabaci. Onion seedlings (Allium cepa L. cv. Giza 20) were sown in December $14^{\text {th }}, 2019$ and December $18^{\text {th }}, 2020$. An area of $\left(4200 \mathrm{~m}^{2}\right)$ was divided into equal plots of $60 \mathrm{~m}^{2}(10 \times$ $6)$. Eleven treatments (ten insecticides and control) were distributed using a randomized complete block design (CRBD) with 55 replicates (five replicates for each treatment). Before the start of the experiment, the experiment area did not treated with any insecticide. The trade name, active ingredient and recommended field rates of the used insecticides are shown in Table (1). The tested compounds were sprayed once on March $20^{\text {th }}$ in both seasons at their label recommended rates at the rate of 200 liter per feddan. A Knapsack sprayer, $\mathrm{CP}^{3}$ was used for spraying with the different treatment. The control plots were sprayed with water only. Randomly five onion plants of each replicate (25 plants from each treatment) were picked and kept in paper bags for the further examination in the laboratory. The number of adult and nymphs T. tabaci were counted and recorded just before spraying with insecticides and after one, four, seven and fourteen days after spraying. The reduction percentages of the thrips population were calculated according to the equation Henderson and Tilton (1955) as follows:

Corrected $\%=(1-(($ ncb*nta $) /($ nca*ntb $))) * 100$

Where:

nta $=$ mean numbers of $T$. tabaci in treatment after application

$\mathrm{ncb}=$ mean number of $T$. tabaci in control before application.

$\mathrm{ntb}=$ mean number of $T$. tabaci in treatment before application

nca $=$ mean number of $T$. tabaci in control after application

Table 1. The active ingredients, trade names, formulations and recommended rates of the used insecticides

\begin{tabular}{|c|c|c|c|}
\hline Trade name & $\begin{array}{l}\text { Active ingredient } \\
\text { (\% and formulation) }\end{array}$ & Manufacturer & Recommended rates \\
\hline Actara $^{\circledR}$ & Thiamethoxam 25\% WDG & Syngenta Agro & $50 \mathrm{mg} \mathrm{L}^{-1}$ \\
\hline Confidor $^{\circledR}$ & Imidacloprid 20\% SL & Bayer CropScience & $200 \mathrm{ml} /$ Fed \\
\hline Radiant ${ }^{\circledR}$ & Spinetoram $120 \mathrm{SC}$ & $\begin{array}{l}\text { Dow AgroSciences, LLC, } \\
\text { Indianapolis, IN }\end{array}$ & $120 \mathrm{mi} / 200 \mathrm{~L}$ \\
\hline Admiral $^{\circledR}$ & Pyriproxyfen $10 \%$ EC & Sumitomo Chemical Co. Ltd & $75 \mathrm{ml} / 100$ liter \\
\hline $\begin{array}{l}\text { Malathion }^{\circledR} \\
\text { Pleo }^{\circledR}\end{array}$ & $\begin{array}{l}\text { Malathon 57\% EC } \\
\text { Pyridalyl (50\% EC) }\end{array}$ & $\begin{array}{l}\text { Sinochem Ningbo Chemicals } \\
\text { Sumitomo Chemical Co. Ltd }\end{array}$ & $\begin{array}{l}5 \mathrm{ml} / \text { liter } \\
100 \mathrm{ml} / \mathrm{fed}\end{array}$ \\
\hline Lambada $^{\circledR}$ & Lambda-cyhalothrin 5\% EC & DowAgro Sciences Co., Ltd & $100 \mathrm{~cm} / \mathrm{Fed}$ \\
\hline Biover $^{\circledR}$ & $\begin{array}{c}\text { Beauvaria bassiana (Biover } 10 \% \\
\text { WP.) }\end{array}$ & $\begin{array}{l}\text { Plant Protection Institute, } \\
\text { ARC, Giza, Egypt }\end{array}$ & $\begin{array}{l}200 \text { gm. / } \\
100 \text { lit. water }\end{array}$ \\
\hline Nimbecidine $^{\circledR}$ & Azadirachtin $0.03 \%$ EC & Botanical Extract & $5 \mathrm{Cm} 3 \mathrm{~L}^{-1}$ \\
\hline $\operatorname{Ochin}^{\circledR}$ & Dinotefuran $20 \%$ SG & Mitsui Chemicals & $50 \mathrm{mg} \mathrm{L}^{-1}$ \\
\hline
\end{tabular}

*The used recommended rates were determined based on the recommendations of Egyptian Ministry of Agriculture 


\section{Statistical Analysis:}

Statistical analysis was conducted using SAS program (1989). The ANOVA test was used to evaluate the significant differences among onion cultivars and the reduction percentages of different insecticides. The means were separated using Least Significant Differences Test LSD. Also, correlation coefficient (r) was used to determine the relation of different metrological factors and T. tabaci population density.

\section{RESULTS AND DISCUSSION}

\section{Relative susceptibility of certain onion cultivars to} the infestation with onion $T$. tabaci:

The results shown in Figs. 1 and 2 reveals the seasonally mean number of thrips per plant on five onion cultivars. It was found that, more or less all the tested onion cultivars were attacked by the thrips. During the $1^{\text {st }}$ season, 2020, the cultivar Giza 6 was the most susceptible cultivar to the thrips infestation. The maximum mean number of thrips per plant (23.53 individual/plant) was recorded on this cultivar followed by Beheri (19.56), Giza 20 (14.9), White Giza (12.15), Red Giza (10.1). Similar results were obtained during the $2^{\text {nd }}$ season, 2021 whereas the cultivar Red Giza (9.74 individual/plant) was the most resistant variety to infestation with onion thrips followed by White Giza
(11.91), Giza 20 (15.98), Beheri (20.38) and finally the cultivar Giza 6 (25.22). The present results are in agreement with those of Awadalla et. al., (2017) who found highest average numbers of T. tabaci on Giza 6 compared with Giza 20 and Giza Red. Salman (2000) in Upper Egypt studies the relative susceptibility of certain onion varieties to infestation with $T$. tabaci and found that the onion variety shandweel 1 was the most susceptible variety, meanwhile Giza 20 variety was the least. In the same frame, Malik et al. (2004) studied the susceptibility of six onion varieties (Red Creole, Chiltan-89, Local, Sariab Surkh, White Globe and Local Kandhari) for the infestation with onion thrips in Quetta, Pakistan. They found that the Local Kandhari followed by Sariab Surkh were the most susceptible varieties to thrips infestation while Chiltan-89 was the least. The cultivar preference may be attributed the fact that thrips prefer darker colors. Ellis et al. (1996) reported that thrips attracted to those plants which have darker in color. In the same trend, Quratulain et al., (2019) in Islamabad found that the variety Saryab red was relatively susceptible against thrips while Red imposta was relatively resistant with maximum mean population of $52.41 \pm 15.42$ and $37.02 \pm 9.97$ individuals per plant infestation, respectively.

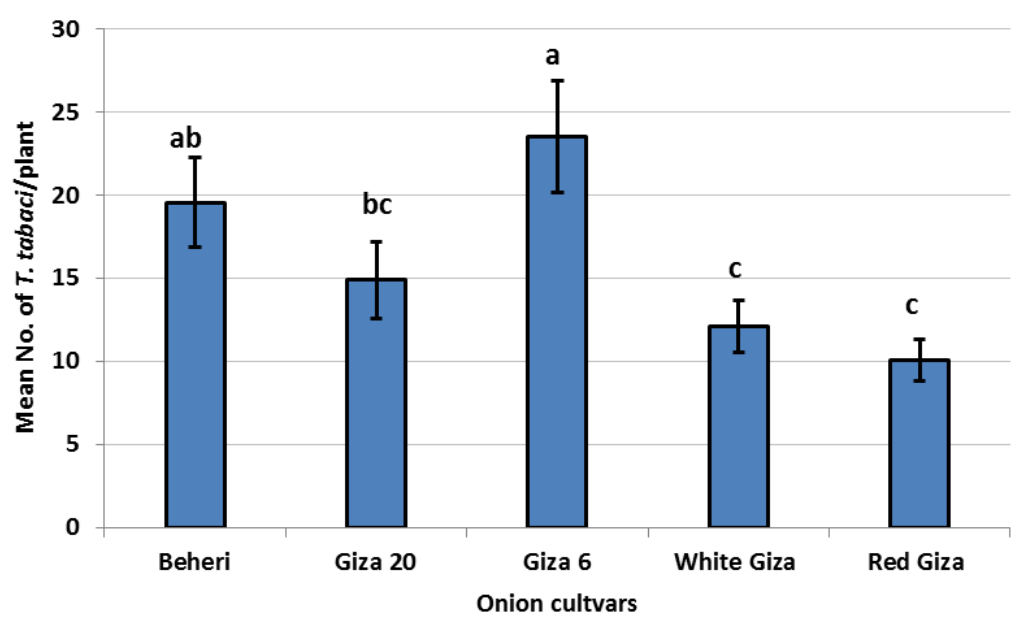

Fig. 1. Susceptibility of different onion cultivars to infestation with onion thrips, $T$. tabaci during the $1^{\text {st }}$ season, 2020 at Beheira Governorate. $(F=5.39$, L.S.D.= 6.64)

Bars with the same letter(s) are not significantly different at $\mathbf{P}<0.05$. 


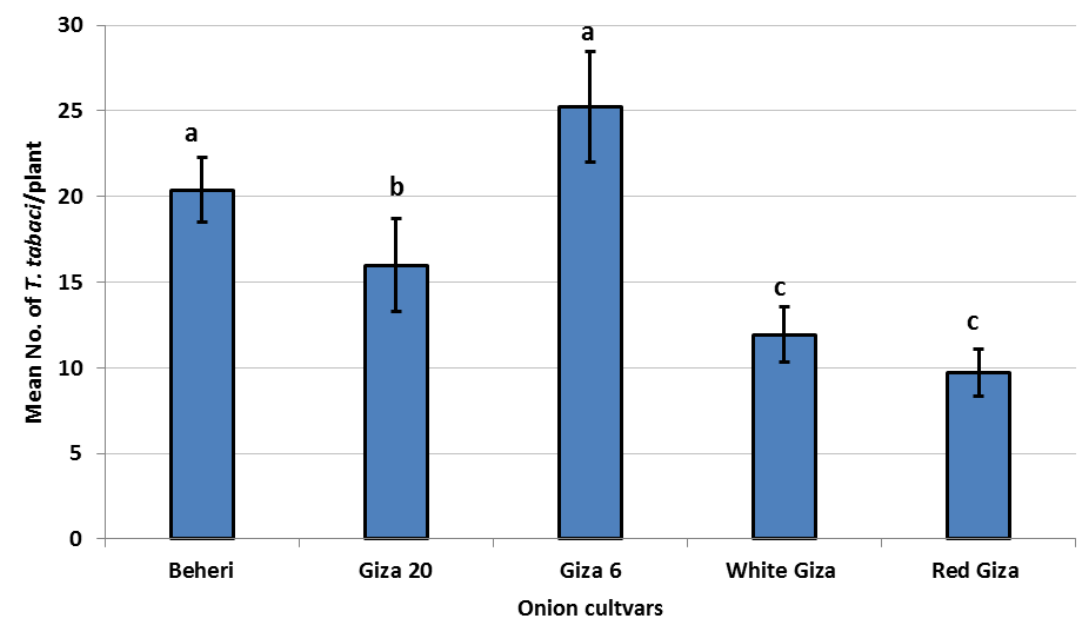

Fig. 2. Susceptibility of different onion cultivars to infestation with onion thrips, T. tabaci during the 2nd season, 2021 at Beheira Governorate. $(F=7.69$, L.S.D.= 6.37)

Bars with the same letter(s) are not significantly different at $P>0.05$.

\section{Efficacy of synthetic chemical and bio-insecticides against onion thrips, $T$. tabaci:}

During the $1^{\text {st }}$ season, 2020 the control efficacy of spinetoram and pyridalyl was significantly higher than that of the other tested insecticides against the nymphs population of $T$. tabaci (as shown in Table 2). The reduction percentages of spinetoram after 1, 4, 7 and 14 days from treatment were $98.48,96.82,93.87$ and 86.57 $\%$, respectively with a general mean of $93.94 \%$. The insecticides, pyridalyl, achieved 94.49, 96.21, 91.36 and $88.25 \%$ after $1,4,7$ and 14 days from treatment with a general mean of $92.58 \%$. According to the statistical analysis, imidacloprid came in the third rank with reduction of $94.93,92.42,86.95$ and $80.62 \%$ after 1day, 4 days, 7 days and 2 weeks, respectively with a general mean of $88.73 \%$. Thiamethoxam came in the fourth rank with reduction of 90.46 (after 1 day), 88.44 (after 4 day), 83.86 (after 1 week) and $73.06 \%$ (after 2 weeks) with a general mean of $83.96 \%$. The reduction percentages of nymphs of $T$. tabaci recorded 89.28, $86.7,72.83$ and 60.15 by using the insecticide lambdacyhalothrin and $82.74,78.51,69.24$ and 63.65 by using dinotefuran after 1, 4, 7 and 14 days, respectively with a general mean of 77.24 for lambda-cyhalothrin and 73.54 $\%$ for dinotefuran. The insect growth regulator, pyriproxyfen came in the seven rank $(83.05,74.17$, 63.32 and $51.53 \%$ after $1,3,4$ and 14 days with a general mean of $68.02 \%$ ) followed by malathion, azadirachtin and finally Beauvaria bassiana with general means of 59.51, 50.88 and $49.87 \%$, respectively.

Concerning the potency of the same tested insecticides against the adults of T. tabaci (Table 3), the highest reduction percentages were achieved by pyridalyl (91.24\%), spinetoram (84.68\%), thiamethoxam $(83.51 \%)$, imidacloprid $(82.37 \%)$, lambda-cyhalothrin (72.19), dinotefuran (69.47 \%) malathion $(65.87 \%)$, pyriproxyfen $(61.84 \%)$, biover (Beauvaria bassiana) (56.06 \%) and azadirachtin (45.74\%).

During the $2^{\text {nd }}$ season, results shown in Tables (4) revealed that, all the tested insecticides achieved considerable reduction percentages in $T$. tabaci nymphs. spinetoram achieved the highest efficiency, it gave the following reduction percentages $(98.8,97.8,95.86$ and $91.67 \%$ after $1,4,7$ and 14 days, respectively) with general mean $96.03 \%$. The insecticide pyridalyl came in the $2^{\text {nd }}$ rank giving $98.74,97.38,91.93$ and $88.46 \%$ after $1,4,7$ and $14 \%$ days, respectively with a general mean of $94.13 \%$. In the $3^{\text {rd }}$ place come the insecticide imidacloprid which achieved $(93.99,91.25,84.87$ and $82.23 \%$ after $1,4,7$ and 14 days, respectively) followed by thiamethoxam $(91.58,90.3,86.71$ and $79.46 \%$ after $1,4,7$ and 14 days, respectively) with a general mean of $88.09 \%$ and 87.01 for imidacloprid thiamethoxam, respectively. The insecticides lambda-cyhalothrin gave reduction percentages of $89.05,86.24,74.83$ and 68.95 with a general mean of $79.77 \%$ followed by dinotefuran which gave reduction percentages of (89.27, 82.13, 72.74 and $61.48 \%$ after $1,4,7$ and 14 days, respectively) with general mean $76.4 \%$. The reduction percentages of insect growth regulator pyriproxyfen recorded 85.91 (after 1 day), 71.69 (after 4 days), 68.91 (after one week) and 60.65\% (after two weeks). Malathion comes in the eights rank followed by azadirachtin and 
Table 2. Efficacy of ten synthetic and bio-insecticides on the nymphs of $T$. tabaci on onion plants under field conditions during 2020 season

\begin{tabular}{|c|c|c|c|c|c|}
\hline \multirow[t]{2}{*}{ Insecticide } & \multicolumn{4}{|c|}{$\begin{array}{c}\text { Days post treatment } \\
\text { Mean No. of nymphs / plant (Reduction \%) }\end{array}$} & \multirow[t]{2}{*}{ General mean } \\
\hline & 1 & 4 & 7 & 14 & \\
\hline Control & $(39.8)$ & $(44.16)$ & $(42.24)$ & $(45.24)$ & \\
\hline Pyriproxyfen & $\begin{array}{c}(6.6) \\
83.05 \pm 3.9^{c}\end{array}$ & $\begin{array}{c}(11.04) \\
74.17 \pm 5.43^{\mathrm{c}}\end{array}$ & $\begin{array}{c}(15.04) \\
63.32 \pm 3.45^{\mathrm{c}}\end{array}$ & $\begin{array}{c}(21.28) \\
51.53 \pm 4.55^{\mathrm{e}}\end{array}$ & $68.02 \pm 12.77^{d}$ \\
\hline Pyridalyl & $\begin{array}{c}(2.28) \\
94.49 \pm 1.29^{\mathrm{ab}}\end{array}$ & $\begin{array}{c}(1.76) \\
96.21 \pm 1.78^{\mathrm{a}}\end{array}$ & $\begin{array}{c}(3.68) \\
91.36 \pm 2.56^{\mathbf{a b}}\end{array}$ & $\begin{array}{c}(5.4) \\
88.25 \pm 2.68^{\mathrm{a}}\end{array}$ & $92.58 \pm 3.7^{\mathrm{a}}$ \\
\hline Malathion & $\begin{array}{c}(11.68) \\
69.36 \pm 7.65^{d}\end{array}$ & $\begin{array}{c}(14.72) \\
65.56 \pm 6.65^{d}\end{array}$ & $\begin{array}{c}(19.8) \\
51.91 \pm 4.08^{d}\end{array}$ & $\begin{array}{l}(20.88) \\
51.2 \pm 14.44^{\mathrm{e}}\end{array}$ & $59.51 \pm 11.73^{\mathrm{e}}$ \\
\hline Lambda-cyhalothrin & $\begin{array}{c}(4.24) \\
89.28 \pm 1.5^{b}\end{array}$ & $\begin{array}{c}(5.84) \\
86.7 \pm 2.83^{\mathbf{b}}\end{array}$ & $\begin{array}{c}(11.4) \\
72.83 \pm 10.81^{\mathrm{c}}\end{array}$ & $\begin{array}{c}(17.84) \\
60.15 \pm 5.68^{\text {de }}\end{array}$ & $77.24 \pm 13.31^{\mathrm{c}}$ \\
\hline Spinetoram & $\begin{array}{c}(0.64) \\
98.48 \pm 1.44^{\mathrm{a}}\end{array}$ & $\begin{array}{c}(1.04) \\
96.82 \pm 1.85^{\mathrm{a}}\end{array}$ & $\begin{array}{c}(2.68) \\
93.87 \pm 1.59^{\mathrm{a}}\end{array}$ & $\begin{array}{c}(6.24) \\
86.57 \pm 2.65^{\mathrm{a}}\end{array}$ & $93.94 \pm 5.01^{\mathrm{a}}$ \\
\hline Azadirachtin & $\begin{array}{c}(16.24) \\
60.79 \pm 7.06^{\mathrm{e}}\end{array}$ & $\begin{array}{c}(19.84) \\
57.1 \pm 5.99^{\mathrm{e}}\end{array}$ & $\begin{array}{c}(23.8) \\
46.08 \pm 7.614^{\text {de }}\end{array}$ & $\begin{array}{c}(28.52) \\
39.53 \pm 9.91^{\mathrm{f}}\end{array}$ & $50.88 \pm 11.27^{\mathrm{f}}$ \\
\hline Thiamethoxam & $\begin{array}{c}(3.92) \\
90.46 \pm 4.1^{\mathbf{b}}\end{array}$ & $\begin{array}{c}(5.36) \\
88.44 \pm 3.15^{\mathbf{b}}\end{array}$ & $\begin{array}{c}(6.88) \\
83.86 \pm 8.34^{\mathbf{b}}\end{array}$ & $\begin{array}{c}(12.6) \\
73.06 \pm 7.53^{\mathbf{b c}}\end{array}$ & $83.96 \pm 8.94^{\mathbf{b}}$ \\
\hline Imidacloprid & $\begin{array}{c}(2.08) \\
94.93 \pm 3.423^{\mathrm{ab}}\end{array}$ & $\begin{array}{c}(3.44) \\
92.42 \pm 3.78^{\mathbf{a b}}\end{array}$ & $\begin{array}{c}(5.68) \\
86.95 \pm 3.06^{\mathbf{a b}}\end{array}$ & $\begin{array}{c}(9.08) \\
80.62 \pm 2.9 \mathrm{a}^{\mathrm{b}}\end{array}$ & $88.73 \pm 6.41^{\mathbf{a b}}$ \\
\hline Beauvaria bassiana & $\begin{array}{c}(14.24) \\
63.54 \pm 6.65^{\mathrm{de}}\end{array}$ & $\begin{array}{c}(18.28) \\
58.45 \pm 4.22^{\mathrm{e}}\end{array}$ & $\begin{array}{c}(25.12) \\
38.5 \pm 16.14^{\mathrm{e}}\end{array}$ & $\begin{array}{c}(27.04) \\
38.98 \pm 11.28^{\mathrm{f}}\end{array}$ & $49.87 \pm 15.11^{\mathrm{f}}$ \\
\hline Dinotefuran & $\begin{array}{c}(7.04) \\
82.74 \pm 4.26^{\mathbf{c}}\end{array}$ & $\begin{array}{c}(9.64) \\
78.51 \pm 6.35^{\mathbf{c}}\end{array}$ & $\begin{array}{c}(13.48) \\
69.24 \pm 3.45^{\mathrm{c}}\end{array}$ & $\begin{array}{c}(16.72) \\
63.65 \pm 7.68^{c d}\end{array}$ & $73.54 \pm 9.31^{\mathrm{cd}}$ \\
\hline F value & 41.993 & 55.049 & 33.998 & 26.745 & 50.064 \\
\hline L.S.D. & 6.002 & 5.81 & 9.597 & 10.11 & 6.48 \\
\hline
\end{tabular}

The reduction percentages followed by the same letter(s) in the column are not significantly different $(\mathrm{P}<0.05)$.

Beauvaria bassiana with a general means of 57.05, 55.77 and $47.42 \%$, respectively. Concerning the efficiency of the insecticides against the adults of $T$. tabaci, the tested insecticides can be arranged according to the general means of reduction percentage in a descending order as follows pyridalyl, spinetoram, thiamethoxam, imidacloprid, lambda-cyhalothrin, dinotefuran, pyriproxyfen, malathion, Beauvaria bassiana and azadirachtin with general means of 95.16, $93.65,89.75,80.38,68.69,65.9,63.81,63.37,58.28$ and $51.86 \%$, respectively (Table 5 ).

From the current experiments, it appears that spinetoram and pyridalyl were the most effective compounds tested to control T. tabaci. These results are in harmony with the results of Khaliq et al. (2014);
Srivastava et al. (2014); Marasigan et al. (2016) and Renkema, et al. (2018) who stated that spinetoram is an effective insecticide for control of different thrips species in a different variety of crops, providing rapidknockdown and sustained suppression of populations. According to Gholami and Sadeghi, (2016) each of spinosad, pyridalyl, and azadirachtin are new and effective insecticides for the control of western flower thrips in vegetable greenhouses. In this study, imidacloprid was highly effective and had a strong residual activity during the two seasons which gave a significant reduction to nymphs and adults population of T. tabaci population after $1,7,10$ and 14 day after treatment compared to the control. Similar results were obtained by Omar and El-Kholy (2001) who compared 
bioefficacy of some traditional and non-traditional insecticides against $T$. tabaci on onion plants, they found imidacloprid gave the highest reduction percentages of the onion thrips. In our experiments the bioinsecticide, Beauvaria bassiana was the least effective insecticides against the T. tabaci (nymphs) in agreement with the results of Mahmoud, (2011) who studied efficacy of some chemical (actellic and marsal) and non-chemical (Beauvaria bassiana) insecticides against onion thrips and found that Biover 10\% WP was the lowest effectiveness. Also, the current results support the results of Zepa, et al. (2011) who found that the most efficient in controlling $T$. tabaci were the products Actara (thiamethoxam) and Confidor Energy (imidacloprid) which having an efficiency of over $97 \%$. On the other hand, Sallam et al. (2018) studied the efficacy of certain insecticides against onion thrips under field conditions compared to selected alternatives. They found that the lowest thrips reduction percentages were observed in plots treated with azadirachtin and the highest reduction percentages were observed in plots treated methomyl.

Table 3. Efficacy of ten synthetic and bio-insecticides on the adults of $T$. tabaci on onion plants under field conditions during 2020 season

\begin{tabular}{|c|c|c|c|c|c|}
\hline \multirow{2}{*}{ Insecticides } & \multicolumn{4}{|c|}{$\begin{array}{c}\text { Days post treatment } \\
\text { Mean No. of adults / plant (Reduction \%) }\end{array}$} & \multirow{2}{*}{ General mean } \\
\hline & 1 & 4 & 7 & 14 & \\
\hline Control & (10) & (8) & (11.6) & (13.08) & \\
\hline Pyriproxyfen & $\begin{array}{c}(2.96) \\
73.59 \pm 12.91^{\text {de }}\end{array}$ & $\begin{array}{c}(2.36) \\
62.11 \pm 13.51^{\mathrm{de}}\end{array}$ & $\begin{array}{c}(3.84) \\
57.34 \pm 23.58^{\mathrm{de}}\end{array}$ & $\begin{array}{c}(4) \\
54.3 \pm 21.69^{\text {de }}\end{array}$ & $61.84 \pm 18.6 \mathrm{~d}^{\mathrm{e}}$ \\
\hline Pyridalyl & $\begin{array}{c}(0.92) \\
95.95 \pm 6.81^{\mathrm{a}}\end{array}$ & $\begin{array}{c}(1.08) \\
92.55 \pm 10.31^{\mathrm{a}}\end{array}$ & $\begin{array}{c}(1.92) \\
91.33 \pm 4.97^{\mathrm{a}}\end{array}$ & $\begin{array}{c}(1.84) \\
85.12 \pm 7.1^{\mathrm{a}}\end{array}$ & $91.24 \pm 8.01^{\mathrm{a}}$ \\
\hline Malathion & $\begin{array}{c}(2.36) \\
68.55 \pm 5.99^{\mathrm{ef}}\end{array}$ & $\begin{array}{c}(2.56) \\
66.52 \pm 15.71^{\mathrm{cd}}\end{array}$ & $\begin{array}{c}(3.96) \\
61.95 \pm 17.5^{\text {cde }}\end{array}$ & $\begin{array}{c}(4.96) \\
66.48 \pm 10.23^{\text {bcd }}\end{array}$ & $65.87 \pm 12.34^{\mathrm{cd}}$ \\
\hline $\begin{array}{l}\text { Lambda- } \\
\text { cyhalothrin }\end{array}$ & $\begin{array}{c}(1.68) \\
82.67 \pm 7.88^{\text {bcd }}\end{array}$ & $\begin{array}{c}(1.76) \\
76.2 \pm 10.22^{\text {bc }}\end{array}$ & $\begin{array}{c}(3.84) \\
63.14 \pm 17.43^{\text {cd }}\end{array}$ & $\begin{array}{c}(3.96) \\
66.51 \pm 15.42^{\text {bcd }}\end{array}$ & $72.19 \pm 14.57^{\mathrm{c}}$ \\
\hline Spinetoram & $\begin{array}{c}(0.28) \\
88.54 \pm 9.59^{\text {abc }}\end{array}$ & $\begin{array}{c}(0.44) \\
85.07 \pm 5.97^{\mathrm{ab}}\end{array}$ & $\begin{array}{c}(0.8) \\
81.6 \pm 6.86^{\mathrm{a}}\end{array}$ & $\begin{array}{c}(1.68) \\
83.5 \pm 8.1^{\mathrm{ab}}\end{array}$ & $84.68 \pm 7.58 \mathrm{a}^{\mathrm{b}}$ \\
\hline Azadirachtin & $\begin{array}{c}(3.88) \\
61.34 \pm 11.77^{\mathrm{f}}\end{array}$ & $\begin{array}{c}(4.08) \\
49.59 \pm 8.38^{\mathrm{e}}\end{array}$ & $\begin{array}{c}(7.16) \\
38.66 \pm 13.26^{\mathrm{f}}\end{array}$ & $\begin{array}{c}(8.92) \\
33.35 \pm 10.22^{\mathrm{f}}\end{array}$ & $45.74 \pm 14.98^{f}$ \\
\hline Thiamethoxam & $\begin{array}{c}(0.6) \\
94.25 \pm 4.4^{\mathrm{ab}}\end{array}$ & $\begin{array}{c}(1.36) \\
86.06 \pm 4.56^{\mathrm{ab}}\end{array}$ & $\begin{array}{c}(2.64) \\
78.54 \pm 13.48^{\mathrm{abc}}\end{array}$ & $\begin{array}{c}(3.4) \\
75.19 \pm 11.54^{\mathrm{abc}}\end{array}$ & $83.51 \pm 11.47^{\mathrm{ab}}$ \\
\hline Imidacloprid & $\begin{array}{c}(1.2) \\
90.51 \pm 5.93^{\text {abc }}\end{array}$ & $\begin{array}{c}(1.4) \\
86.89 \pm 6.39^{\mathrm{ab}}\end{array}$ & $\begin{array}{c}(2.68) \\
80.82 \pm 6.29^{\mathrm{ab}}\end{array}$ & $\begin{array}{c}(4.32) \\
71.27 \pm 14.92^{\mathrm{abcd}}\end{array}$ & $82.37 \pm 11.27^{\mathbf{b}}$ \\
\hline $\begin{array}{l}\text { Beauvaria } \\
\text { bassiana }\end{array}$ & $\begin{array}{c}(3.04) \\
62.58 \pm 15.07^{\text {ef }}\end{array}$ & $\begin{array}{c}(2.12) \\
69.94 \pm 12.13^{\text {cd }}\end{array}$ & $\begin{array}{c}(5.64) \\
44.68 \pm 13.038^{\mathrm{ef}}\end{array}$ & $\begin{array}{c}(5.8) \\
47.06 \pm 19.02^{\mathrm{ef}}\end{array}$ & $56.06 \pm 17.55^{\mathrm{e}}$ \\
\hline Dinotefuran & $\begin{array}{c}(1.92) \\
81.32 \pm 8.37^{\mathrm{cd}}\end{array}$ & $\begin{array}{c}(2.16) \\
73.78 \pm 10.51^{\text {bcd }}\end{array}$ & $\begin{array}{c}(4.4) \\
63.57 \pm 9.58^{\text {bcd }}\end{array}$ & $\begin{array}{c}(5.64) \\
59.22 \pm 8.82^{\text {cde }}\end{array}$ & $69.47 \pm 12.34^{\mathrm{cd}}$ \\
\hline F value & 9.165 & 8.270 & 7.524 & 7.170 & 23.241 \\
\hline L.S.D. & 12.08 & 13.19 & 17.38 & 17.28 & 8.31 \\
\hline
\end{tabular}

The reduction percentages followed by the same letter(s) in the column are not significantly different $(\mathrm{P}<0.05)$. 
Table 4. Efficacy of ten synthetic and bio-insecticides on the nymphs of T. tabaci on onion plants under field conditions during 2021 season

\begin{tabular}{|c|c|c|c|c|c|}
\hline \multirow[t]{2}{*}{ Insecticide } & \multicolumn{4}{|c|}{$\begin{array}{l}\text { Days post treatment } \\
\text { Mean No. of nymphs / plant (Reduction \%) }\end{array}$} & \multirow[t]{2}{*}{ General mean } \\
\hline & 1 & 4 & 7 & 14 & \\
\hline Control & (33.68) & $(36.4)$ & (35.28) & $(37.8)$ & \\
\hline Pyriproxyfen & $\begin{array}{c}(4.52) \\
85.91 \pm 2.66^{\mathbf{c}}\end{array}$ & $\begin{array}{c}(9.82) \\
71.69 \pm 6.09^{d}\end{array}$ & $\begin{array}{c}(10.4) \\
68.91 \pm 4.35^{\mathrm{c}}\end{array}$ & $\begin{array}{c}(14.08) \\
60.65 \pm 6.06^{c}\end{array}$ & $71.79 \pm 10.41^{\mathrm{e}}$ \\
\hline Pyridalyl & $\begin{array}{c}(0.44) \\
98.74 \pm 2.07^{\mathrm{a}}\end{array}$ & $\begin{array}{c}(0.88) \\
97.38 \pm 2.88^{\mathrm{a}}\end{array}$ & $\begin{array}{c}(1.56) \\
91.93 \pm 5.73^{\text {ab }}\end{array}$ & $\begin{array}{c}(3.16) \\
88.46 \pm 6.84^{\text {ab }}\end{array}$ & $94.13 \pm 6.13^{\mathrm{ab}}$ \\
\hline Malathion & $\begin{array}{c}(10.08) \\
68.53 \pm 3.89^{d}\end{array}$ & $\begin{array}{c}(13.16) \\
62.05 \pm 2.78^{\mathrm{e}}\end{array}$ & $\begin{array}{c}(15.24) \\
54.53 \pm 8.53^{\mathrm{d}}\end{array}$ & $\begin{array}{c}(20.28) \\
43.1 \pm 9.68^{d}\end{array}$ & $57.05 \pm 11.58^{\mathrm{f}}$ \\
\hline Lambda-cyhalothrin & $\begin{array}{c}(3.48) \\
89.05 \pm 6.36^{\mathbf{b c}}\end{array}$ & $\begin{array}{c}(4.8) \\
86.24 \pm 4.77^{\mathbf{b c}}\end{array}$ & $\begin{array}{c}(8.52) \\
74.83 \pm 6.73^{c}\end{array}$ & $\begin{array}{c}(11.08) \\
68.95 \pm 7.74^{\mathrm{c}}\end{array}$ & $79.77 \pm 10.31^{d}$ \\
\hline Spinetoram & $\begin{array}{c}(0.4) \\
98.8 \pm 1.83^{\mathrm{a}}\end{array}$ & $\begin{array}{c}(0.96) \\
97.8 \pm 2.31^{a}\end{array}$ & $\begin{array}{c}(3.04) \\
95.86 \pm 2.54^{\mathrm{a}}\end{array}$ & $\begin{array}{c}(4.4) \\
91.67 \pm 4.46^{\mathrm{a}}\end{array}$ & $96.03 \pm 3.9^{\mathrm{a}}$ \\
\hline Azadirachtin & $\begin{array}{c}(10.76) \\
68.23 \pm 8.97^{d}\end{array}$ & $\begin{array}{c}(14.28) \\
60.68 \pm 7.23^{\mathrm{e}}\end{array}$ & $\begin{array}{c}(15.6) \\
56.14 \pm 6.8^{d}\end{array}$ & $\begin{array}{c}(23.52) \\
38.03 \pm 8.99^{d}\end{array}$ & $55.77 \pm 13.59^{f}$ \\
\hline Thiamethoxam & $\begin{array}{c}(2.16) \\
91.58 \pm 7 \mathrm{ab}^{\mathrm{c}}\end{array}$ & $\begin{array}{c}(3.2) \\
90.3 \pm 4.93^{\mathrm{ab}}\end{array}$ & $\begin{array}{c}(5.36) \\
86.71 \pm 4.45^{\mathbf{b}}\end{array}$ & $\begin{array}{c}(6.76) \\
79.46 \pm 4.1^{b}\end{array}$ & $87.01 \pm 6.82^{c}$ \\
\hline Imidacloprid & $\begin{array}{c}(2.92) \\
93.99 \pm 3.73^{\text {ab }}\end{array}$ & $\begin{array}{c}(3.72) \\
91.25 \pm 4.99^{\mathbf{a b}}\end{array}$ & $\begin{array}{c}(4.76) \\
84.87 \pm 5.11^{b}\end{array}$ & $\begin{array}{c}(8.08) \\
82.23 \pm 5.35^{\mathbf{b}}\end{array}$ & $88.09 \pm 6.58^{\mathbf{b c}}$ \\
\hline Beauvaria bassiana & $\begin{array}{c}(13.12) \\
60.43 \pm 10.28^{\mathrm{e}}\end{array}$ & $\begin{array}{c}(17.04) \\
51.99 \pm 13.27^{\mathrm{f}}\end{array}$ & $\begin{array}{c}(20.36) \\
41.15 \pm 10.21^{\mathrm{e}}\end{array}$ & $\begin{array}{c}(23.88) \\
36.09 \pm 9.15^{\mathrm{d}}\end{array}$ & $47.42 \pm 13.9^{\mathrm{g}}$ \\
\hline Dinotefuran & $\begin{array}{c}(3.8) \\
89.27 \pm 4.33^{\mathbf{b c}}\end{array}$ & $\begin{array}{c}(6.68) \\
82.13 \pm 6.84^{\mathrm{c}}\end{array}$ & $\begin{array}{c}(10.2) \\
72.74 \pm 7.2^{\mathrm{c}}\end{array}$ & $\begin{array}{c}(15.4) \\
61.48 \pm 7.5^{\mathrm{c}}\end{array}$ & $76.4 \pm 12.29 \mathrm{~d}^{\mathrm{e}}$ \\
\hline F value & 27.795 & 33.375 & 37.555 & 40.913 & 56.870 \\
\hline L.S.D. & 7.43 & 8.14 & 8.33 & 9.25 & 6.3 \\
\hline
\end{tabular}

The reduction percentages followed by the same letter(s) in the column are not significantly different $(\mathrm{P}<0.05)$. 
Table 5. Efficacy of ten synthetic and bio-insecticides on the adults of $T$. tabaci on onion plants under field conditions during 2021 season

\begin{tabular}{|c|c|c|c|c|c|}
\hline \multirow{2}{*}{ Insecticide } & \multicolumn{4}{|c|}{$\begin{array}{l}\text { Days post treatment } \\
\text { Mean No. of adults / plant (Reduction \%) }\end{array}$} & \multirow{2}{*}{ General mean } \\
\hline & 1 & 4 & 7 & 14 & \\
\hline Control & $(4.16)$ & $(3.16)$ & (5) & $(5.64)$ & \\
\hline Pyriproxyfen & $\begin{array}{c}(1.18) \\
70.38 \pm 9.87^{\text {cd }}\end{array}$ & $\begin{array}{c}(1.12) \\
64.49 \pm 6.09^{\mathbf{c}}\end{array}$ & $\begin{array}{c}(1.88) \\
63.25 \pm 6.37^{\mathrm{cd}}\end{array}$ & $\begin{array}{c}(2.36) \\
57.12 \pm 5.92^{\mathrm{c}}\end{array}$ & $63.81 \pm 8.22^{\mathrm{cd}}$ \\
\hline Pyridalyl & $\begin{array}{c}(0.08) \\
97.82 \pm 4.87^{\mathrm{a}}\end{array}$ & $\begin{array}{c}(0.08) \\
97.54 \pm 3.37^{\mathrm{a}}\end{array}$ & $\begin{array}{c}(0.28) \\
94.49 \pm 5.7^{\mathrm{a}}\end{array}$ & $\begin{array}{c}(0.6) \\
90.8 \pm 3.31^{\mathbf{a}}\end{array}$ & $95.16 \pm 5^{\mathrm{a}}$ \\
\hline Malathion & $\begin{array}{c}(0.96) \\
71.93 \pm 5.02^{\text {bed }}\end{array}$ & $\begin{array}{c}(1) \\
64.64 \pm 6.22^{\mathrm{c}}\end{array}$ & $\begin{array}{c}(1.68) \\
59.42 \pm 14.41^{\text {cd }}\end{array}$ & $\begin{array}{c}(2.12) \\
57.48 \pm 6.88^{c}\end{array}$ & $63.37 \pm 10^{\text {cd }}$ \\
\hline Lambda-cyhalothrin & $\begin{array}{c}(0.8) \\
78.77 \pm 7.95^{\text {bc }}\end{array}$ & $\begin{array}{c}(0.96) \\
69.69 \pm 6.76^{\mathrm{c}}\end{array}$ & $\begin{array}{c}(1.64) \\
67.65 \pm 3.5^{\mathbf{b c}}\end{array}$ & $\begin{array}{c}(2.24) \\
58.65 \pm 11.97^{\mathrm{c}}\end{array}$ & $68.69 \pm 10.46^{\mathbf{c}}$ \\
\hline Spinetoram & $\begin{array}{c}(0.08) \\
97.75 \pm 5.02^{\mathrm{a}}\end{array}$ & $\begin{array}{c}(0.08) \\
96.26 \pm 8.35^{\mathrm{a}}\end{array}$ & $\begin{array}{c}(0.24) \\
91.89 \pm 9.13^{\mathrm{a}}\end{array}$ & $\begin{array}{c}(0.44) \\
88.68 \pm 5.8^{\mathrm{a}}\end{array}$ & $93.65 \pm 7.63^{\mathrm{a}}$ \\
\hline Azadirachtin & $\begin{array}{c}(1.28) \\
64.65 \pm 7.56^{\mathrm{d}}\end{array}$ & $\begin{array}{c}(1.08) \\
52.89 \pm 7.37^{\mathrm{d}}\end{array}$ & $\begin{array}{c}(2.32) \\
49.5 \pm 4.77^{\mathrm{e}}\end{array}$ & $\begin{array}{c}(3.44) \\
40.41 \pm 6^{\mathrm{d}}\end{array}$ & $51.86 \pm 10.73^{\mathrm{e}}$ \\
\hline Thiamethoxam & $\begin{array}{c}(0.28) \\
94.21 \pm 3.98^{\mathrm{a}}\end{array}$ & $\begin{array}{c}(0.28) \\
92.38 \pm 2.59^{\mathrm{ab}}\end{array}$ & $\begin{array}{c}(0.76) \\
89.28 \pm 3.26^{\mathrm{a}}\end{array}$ & $\begin{array}{c}(1.16) \\
83.15 \pm 4.53^{\mathrm{a}}\end{array}$ & $89.75 \pm 5.47^{\mathrm{a}}$ \\
\hline Imidacloprid & $\begin{array}{c}(0.6) \\
89.67 \pm 6.52^{\mathrm{a}}\end{array}$ & $\begin{array}{c}(0.72) \\
83.93 \pm 6.72^{\mathbf{b}}\end{array}$ & $\begin{array}{c}(1.48) \\
76.7 \pm 6.72^{b}\end{array}$ & $\begin{array}{c}(2.04) \\
71.22 \pm 10.11^{\mathbf{b}}\end{array}$ & $80.38 \pm 10.06^{\mathbf{b}}$ \\
\hline Beauvaria bassiana & $\begin{array}{c}(1.16) \\
67.82 \pm 8.19^{d}\end{array}$ & $\begin{array}{c}(1.32) \\
68.38 \pm 10.89^{c}\end{array}$ & $\begin{array}{c}(2.36) \\
55.66 \pm 4.47^{\text {de }}\end{array}$ & $\begin{array}{c}(2.96) \\
41.27 \pm 4.21^{\mathrm{d}}\end{array}$ & $58.28 \pm 13.26^{\mathrm{d}}$ \\
\hline Dinotefuran & $\begin{array}{c}(0.84) \\
80.23 \pm 6.31^{\text {b }}\end{array}$ & $\begin{array}{c}(1.08) \\
68.35 \pm 8.26^{c}\end{array}$ & $\begin{array}{c}(2.28) \\
59.57 \pm 6.44^{\text {cd }}\end{array}$ & $\begin{array}{c}(2.8) \\
55.45 \pm 6.95^{\mathrm{c}}\end{array}$ & $65.9 \pm 11.68^{c}$ \\
\hline F value & 17.665 & 24.185 & 25.693 & 33.760 & 52.291 \\
\hline L.S.D. & 8.64 & 8.998 & 9.185 & 8.99 & 5.98 \\
\hline
\end{tabular}

The reduction percentages followed by the same letter(s) in the column are not significantly different $(\mathrm{P}<0.05)$.

\section{Population dynamic of onion thrips:}

The onion thrips, T. tabaci remained a regular pest on onion plants during the growing season. The population buildup started from the $2^{\text {nd }}$ week of January and remained active up to the $2^{\text {nd }}$ week of May. Data shown in Table 6 and 7 revealed that $T$. tabaci first observed on $11^{\text {th }}$ January 2020 (2.53 individuals / plant) and on $9^{\text {th }}$ January 2021 (2.48 individuals / plant). In February the population of onion thrips were increased from 6.76 individuals/ plant to 19.89) and from 10.88 to 17.76 in the $1^{\text {st }}$ and $2^{\text {nd }}$ season, respectively. The first population peek was recorded on $3^{\text {rd }}$ week of March with average of 30.19 individuals plant ${ }^{-1}$ in the $1^{\text {st }}$ season (2020) and in mid-March with average of 24.81 individuals plant ${ }^{-1}$ in the $2^{\text {nd }}$ season (2021). Later, as the onion crop started to mature, the population dropped to 8.44 thrips /plant in the 2020 and 10.04 thrips /plant in 2021 towards the end of May. The current results support the results of Darwish, 2015 and El-Sheikh, 2017 who found that in January and February the population density of onion thrips was relatively with low numbers. The population density increased rapidly to reach maximum abundance in April. The results of the population trends of nymphs and adults of T. tabaci revealed that the insect had two generations during the onion growing period. 
Table 6. The population density of onion thrips, T. tabaci (mean /plant) infesting onion plants during the $1^{\text {st }}$ season, 2020 at Nobaria district in relation to abiotic factors

\begin{tabular}{|c|c|c|c|c|c|c|c|c|c|}
\hline \multirow{2}{*}{$\begin{array}{c}\text { Investigation } \\
\text { date }\end{array}$} & \multirow[b]{2}{*}{ Nymphs } & \multirow[b]{2}{*}{ Adults } & \multirow{2}{*}{$\begin{array}{c}\text { Total } \\
\text { population }\end{array}$} & \multicolumn{3}{|c|}{ Weekly mean of } & \multicolumn{3}{|c|}{ Weekly mean of } \\
\hline & & & & $\begin{array}{l}\text { Mini. } \\
\text { Temp. }\end{array}$ & $\begin{array}{l}\text { Maxi. } \\
\text { Temp. }\end{array}$ & $\begin{array}{l}\text { Mean } \\
\text { Temp. }\end{array}$ & $\begin{array}{l}\text { Mini. } \\
\text { R.H.\% }\end{array}$ & $\begin{array}{l}\text { Maxi. } \\
\text { R.H.\% }\end{array}$ & $\begin{array}{c}\text { Mean } \\
\text { R.H.\% }\end{array}$ \\
\hline 11-Jan & 1.94 & 0.59 & 2.53 & 17.14 & 10 & 13.57 & 79.5 & 51.4 & 65.45 \\
\hline 18-Jan & 2.88 & 1.31 & 4.19 & 18 & 11.43 & 14.71 & 81.02 & 57.23 & 69.13 \\
\hline 25-Jan & 3.94 & 1.21 & 5.15 & 17.29 & 11.43 & 14.36 & 80.43 & 53.85 & 67.14 \\
\hline 01-Feb & 4.81 & 1.95 & 6.76 & 18.43 & 10.43 & 14.43 & 79.75 & 49.43 & 64.59 \\
\hline 08-Feb & 4.75 & 3.93 & 8.68 & 19.29 & 11.29 & 15.29 & 70.69 & 38.04 & 54.36 \\
\hline $15-\mathrm{Feb}$ & 10.5 & 2.44 & 12.94 & 18.57 & 10.43 & 14.5 & 79.57 & 45.99 & 62.78 \\
\hline $22-\mathrm{Feb}$ & 10.94 & 3.14 & 14.08 & 18.14 & 12.29 & 15.21 & 79.43 & 48.93 & 64.18 \\
\hline 29-Feb & 13.25 & 6.64 & 19.89 & 20.14 & 10.57 & 15.36 & 76.71 & 41.31 & 59.01 \\
\hline 07-Mar & 12.83 & 11 & 23.83 & 21.14 & 10.43 & 15.79 & 78.63 & 39.8 & 59.21 \\
\hline 14-Mar & 18.44 & 8.73 & 27.16 & 20.86 & 12 & 16.43 & 81.01 & 42.94 & 61.98 \\
\hline 21-Mar & 20.06 & 10.13 & 30.19 & 19.57 & 12.71 & 16.14 & 80.67 & 40.6 & 60.64 \\
\hline 28-Mar & 12.875 & 9.2 & 22.08 & 21.29 & 13.43 & 17.36 & 81.3 & 40.43 & 60.86 \\
\hline 04-Apr & 12.31 & 10.21 & 22.53 & 25.71 & 13 & 19.36 & 79.76 & 41.61 & 60.69 \\
\hline 11-Apr & 18.78 & 8.06 & 26.84 & 21 & 13.86 & 17.43 & 80.16 & 43.01 & 61.59 \\
\hline 18-Apr & 14.14 & 9.88 & 24.01 & 23.14286 & 13.57 & 18.36 & 82.74 & 41.53 & 62.14 \\
\hline 25-Apr & 10.31 & 7.11 & 17.43 & 24.14286 & 15.71 & 19.93 & 81.37 & 41.91 & 61.64 \\
\hline 02-May & 6.19 & 6 & 12.19 & 24.71429 & 15 & 19.86 & 83.76 & 49.64 & 66.7 \\
\hline 09-May & 4.38 & 4.06 & 8.44 & 25.42857 & 15.14 & 20.29 & 78.63 & 39.8 & 59.21 \\
\hline
\end{tabular}

Table 7. The population density of onion thrips, T. tabaci (mean /plant) infesting onion plants during the $2^{\text {nd }}$ season, 2021 at Nobaria district in relation to abiotic factors

\begin{tabular}{cccccccccc}
\hline & & & \multicolumn{3}{c}{ Weekly mean of } & \multicolumn{3}{c}{ Weekly mean of } \\
\cline { 5 - 9 } $\begin{array}{c}\text { Investigation } \\
\text { date }\end{array}$ & Nymphs & Adults & $\begin{array}{c}\text { Total } \\
\text { population }\end{array}$ & $\begin{array}{c}\text { Mini. } \\
\text { Temp. }\end{array}$ & $\begin{array}{c}\text { Maxi. } \\
\text { Temp. }\end{array}$ & $\begin{array}{c}\text { Mean } \\
\text { Temp. }\end{array}$ & $\begin{array}{c}\text { Mini. } \\
\text { R.H.\% }\end{array}$ & $\begin{array}{c}\text { Maxi. } \\
\text { R.H.\% }\end{array}$ & $\begin{array}{c}\text { Mean } \\
\text { R.H.\% }\end{array}$ \\
\hline 09-Jan & 1.29 & 1.19 & 2.475 & 21.71 & 12.86 & 17.29 & 80.43 & 52.63 & 66.53 \\
16-Jan & 1.25 & 2.16 & 3.413 & 21.14 & 12.43 & 16.79 & 78.04 & 48.2 & 63.12 \\
23-Jan & 2.54 & 2.44 & 4.975 & 17.29 & 10.57 & 13.93 & 77.03 & 50.53 & 63.78 \\
30-Jan & 2.88 & 5.75 & 8.625 & 19.43 & 10.29 & 14.86 & 77.59 & 50.41 & 64 \\
05-Feb & 3.5 & 7.38 & 10.875 & 22.86 & 12.57 & 17.71 & 75.99 & 42.33 & 59.16 \\
12-Feb & 4.53 & 8.63 & 13.15 & 22 & 10.43 & 16.21 & 76.8 & 43.46 & 60.13 \\
19-Feb & 7.43 & 9.44 & 16.86 & 17 & 8.857 & 12.93 & 74.96 & 38.79 & 56.87 \\
26-Feb & 8 & 9.76 & 17.763 & 18.57 & 11.14 & 14.86 & 77.6 & 44.23 & 60.91 \\
04-Mar & 6.31 & 13.13 & 19.438 & 19.71 & 11.14 & 15.43 & 78.63 & 39.8 & 59.21 \\
11-Mar & 5.88 & 12.69 & 24.813 & 22.71 & 12.86 & 17.79 & 78.1 & 40.37 & 59.24 \\
18-Mar & 5.06 & 17.88 & 22.938 & 21 & 10.43 & 15.71 & 78.93 & 50.07 & 64.5 \\
25-Mar & 7.15 & 15 & 22.15 & 21.29 & 14.43 & 17.86 & 78.14 & 45.89 & 62.01 \\
01-Apr & 5.54 & 18.38 & 23.913 & 20 & 11.57 & 15.79 & 79.97 & 44.04 & 62.01 \\
08-Apr & 10.31 & 21.5 & 31.813 & 23.71 & 13.43 & 18.57 & 80.89 & 45.04 & 62.96 \\
15-Apr & 10.74 & 18.44 & 29.175 & 18.43 & 11.86 & 15.143 & 78.76 & 43.99 & 61.37 \\
22-Apr & 6.56 & 18 & 24.563 & 27.43 & 16.14 & 21.79 & 78.17 & 38.3 & 58.24 \\
29-Apr & 6.06 & 6.59 & 12.65 & 30 & 15.43 & 22.71 & 80.9 & 41.76 & 61.33 \\
06-May & 4.6 & 5.44 & 10.04 & 34.29 & 17.714 & 26 & 78.63 & 39.8 & 59.21 \\
\hline
\end{tabular}


Table 8. The correlation coefficient between different meteorological factors and adult, nymphs and total population of $T$. tabaci

\begin{tabular}{cccccccc}
\hline \multirow{2}{*}{ Seasons } & \multirow{2}{*}{ Stage } & Mini. & Maxi. & Mean & Mini. & Maxi. & Mean \\
& Nemp. & Temp. & Temp. & R.H.\% & R.H.\% & R.H.\% \\
\hline \multirow{2}{*}{2020} & Nymphs & 0.216 & 0.191 & 0.218 & 0.206 & $-0.566^{*}$ & -0.366 \\
& Adults & $0.592^{* *}$ & 0.374 & $0.536^{*}$ & 0.200 & $-0.726^{* *}$ & $-0.495^{*}$ \\
& Total population & 0.461 & 0.454 & $0.484^{*}$ & 0.223 & $-0.551^{*}$ & -0.348 \\
\hline \multirow{2}{*}{2021} & Nymphs & 0.049 & 0.056 & 0.014 & 0.237 & -0.423 & -0.297 \\
& Adults & 0.059 & 0.040 & 0.026 & 0.272 & -0.327 & -0.202 \\
& Total population & 0.040 & 0.065 & 0.005 & 0.154 & $-0.492^{*}$ & -0.385 \\
\hline
\end{tabular}

Significant correlation $(\mathrm{P}<0.05) * *$ highly significant $(\mathrm{P}<0.01)$

\section{Influence of abiotic factors on population dynamics of onion thrips, T. tabaci in onion plants:}

As shown in Table 8 the correlation coefficient between different meteorological factors and adults, nymphs and total population of $T$. tabaci revealed that the onion thrips exhibited a highly significant negative correlation with maximum, mean relative humidity. However, these stages were positively correlated with minimum, maximum and mean of temperature. These results support the results of Gill, et al. (2015) who stated that the dry and hot weather can lead to an increase in the populations of T. tabaci and the severity of thrips injury to onion and other crops. Also, the current results confirm that the nymph stage was more affected by the weather factors than adult stage as indicated by with Hendawy, et al., (2011) and Moraiet and Ansari, (2014). The findings of Hamdy and Salem, (1994) (relatively high temperature have been associated with increase in onion thrips population, while high relative humidity and rainfall reduce thrips population) are consistent to our results.

\section{CONCLUSIONS}

In the light of above mentioned results and numerical data, it is concluded that Red Giza and Giza 6 were the most tolerant and susceptible cultivars against T. tabaci infestation. Also, it is concluded that spinetoram and pyridalyl insecticides approved to be most effective against cotton thrips, $T$. tabaci. The onion thrips had two generations during the onion growing period. There was a significantly negative correlation $(\mathrm{P}<0.05)$ between population density of onion thrips and relative humidity. The results also revealed that the temperature positively and significantly affect $T$. tabaci population in both seasons 2020 and 2021.

\section{REFERENCES}

Afaf M.S. El- Roby. 2018. Evaluation of some plant extracts and chemical pesticides on garlic thrips, Thrips tabaci and associated vegetative growth parameters in garlic fields. Egypt. J. Agric. Res. 96 (1):39-46. DOI: 10.21608/EJAR.2018.129243

Amro, M.A., G.H. Abd- El-Rahim and A.A. Abd- ElRaheem. 2009. Population fluctuation, relative susceptibility and control of Thrips tabaci (Lind.) on some onion and garlic cultivars and strains. Assiut Univ. Bull. Environ. $\quad$ Res. 12(2): 131- 141. https://auber.journals.ekb.eg/article_149557_c4da483353c 82121e7a18cd09420542e.pdf

Ananthakrishnan, T.N. 1973. Thrips: Biology and control. MacMillan, New Delhi, India.

Awadalla S. S., A. A. Taman and A.A. Aboria. 2017. Influence of the different onion varieties on the population density of the main insect pests infesting onion crop in Kafr El-Shekh region. J. Plant Prot. and Path., Mansoura Univ. 8 (8):403 - 406. DOI: 10.21608/JPPP.2017.46356

Boateng C.O., H.F. Schwartz, M.J. Havey and K. Otto. 2014. Evaluation of onion germplasm for resistance to Iris Yellow Spot (Iris yellow spot virus) and onion thrips, Thrips tabaci. Southwest. Entomol. 39:237-260.

https://doi.org/10.3958/059.039.0218

Darwish, A.A.E. 2015. Ecological and behavioral aspects of Thrips tabaci Lindeman (Thysanoptera : Thripidae) on onion plants. J. Plant Prot. and Path., Mansoura Univ.6 (10): 1399 - 1413. DOI: 10.21608/jppp.2015.75335

Diaz-Montano J., M. Fuchs, B. A. Nault, J. Fail and A.M. Shelton. 2011. Onion thrips (Thysanoptera: Thripidae): A global pest of increasing concern in onion. J. Econ. Entomol. 104: 1-13. DOI: 10.1603/EC10269

Ellis B.W., F.M. Bradley and H. Atthowe. 1996. The organic gardener's Handbook of natural insect and disease control: A Complete Problem-Solving Guide to keeping your garden and yard healthy without chemicals: Paperback 544 pages. 
El-Sheikh, M. F. 2017. Effectiveness of Beauveria bassiana (Bals.) Vuill. and Metarhizium anisopliae (Metsch.) (Deuteromycotina: Hyphomycetes) as Biological Control Agents of the Onion Thrips, Thrips tabaci Lind. J. Plant Prot. and Path., Mansoura Univ. 8 (7): 319 - 323. DOI: 10.21608/jppp.2017.46318

Gill, H.K., H. Garg, A.K. Gill, J.L. Gillett-Kaufman and B.A. Nault. 2015. Onion thrips (Thysanoptera: Thripidae) biology, ecology, and management in onion production systems. Pest Manag. Sci. 6: 1-9. https://doi.org/10.1093/jipm/pmv006

Gholam Z. and A. Sadeghi. 2016. Management strategies for western flower thrips in vegetable greenhouses in Iran: a review. Plant Protect. Sci. 52: 87-98. Doi: 10.17221/2/2015-PPS

Hamdy, M. K. and M. Salem. 1994. The effect of plantation dates of onion temperature and relative humidity on the population density of the onion thrips, Thrips tabaci Lind., in Egypt. Ann. Agric. Sci. Cairo. 39: 417-424.

https://agris.fao.org/agrissearch/search.do?recordID=EG9601360

Hendawy, A.S., S.K.M. El-Fakharany and S.A.A. Kassem. 2011. Ecological and toxicological studies on Thrips tabaci Lindeman and associated spiders on onion plantations. Egypt. J. Biol. Pest Control., 21, 2, pp. 337342

Henderson C.F. and F.W. Tilton 1955. Test with acaricides against the brown wheat mite. J. Econ. Ent. 48: 157-161. https://doi.org/10.1093/jee/48.2.157

Khaliq A., A.A. Khan, M. Afzal, H.M. Tahir, A.M. Raza, A.M. Khan. 2014. Field evaluation of selected botanicals and commercial synthetic insecticides against Thrips tabaci Lindeman (Thysanoptera: Thripidae) populations and predators in onion field plots. Crop Protection. 62: 10-15. https://doi.org/10.1016/j.cropro.2014.03.019

Mahmoud, H.H. 2008. Ecological Studies on certain insect pests of onion with special emphasis on the onion bulb fly Eumerus anoenus Loew. Ph.D. Thesis, Fac. Agric., Cairo Unvi., Egypt 125 pp.

Mahmoud, H.H. 2011. Efficacy of some Chemical and NonChemical Insecticides Against Thrips tabaci Lind. in Onion Fields, and Onion Bulb Fly Eumerus amoenus Loew. in Onion Stores. Assiut J. of Agric. Sci. 42 (2): 476-487.

Malik M.F., M. Nawaz and Z. Hafeez. 2003. Evaluation of Promising Onion (Allium cepa) Varieties Against Thrips Infestation in the Agro-Ecosystem of Balochistan, Pakistan - I. Asian Journal of Plant Sci. 2: 716-718. DOI: 10.3923/ajps.2003.716.718

Marasigan K., M. Toews, J. R. Kemerait, M.R. Abney, A. Culbreath, R. Srinivasan. 2016. Evaluation of alternative to carbamate and organophosphate insecticides against thrips and tomato spotted wilt virus in peanut production. Journal of Economic Entomology. 109: 544-557. DOI: 10.1093/jee/tov336

Morse J. G., M. S. Hoddle. 2006. Invasion biology of thrips. Ann. Rev. Entomol. 51: 67-89. https://doi.org/10.1146/annurev.ento.51.110104.151044
Moraiet M.A. and M.S. Ansari. 2014. Population dynamics of onion thrips, Thrips tabaci, on onion cultivars. Journal of Agroecology and Natural Resource Management. 1(3): 141-147.

Nault, B.A. and M.L. Hessney. 2010. Onion thrips control in onion-2009. Arthropod Manag. Trials 35: E13. https://doi.org/10.4182/amt.2010.E13

Omar, B.A. and M. I. El-Kholy. 2001. Comparative bioefficacy of certain traditional and non-traditional insecticides against thrips infesting onion. J. Agric. Sci. Mansoura Univ. 26(4):2373- 2381. https://doi.org/10.1371/journal.pone.0101791

Quratulain1, Ata-ul-Mohsin, N. Muhammad, S. Ghulam, M. K. Rafique and R. Mahmood. 2020. Screening of onion (Allium cepa L.) accessions for susceptibility to Thrips tabaci L. (Thysanoptera: Thripidae) under insecticide-free field conditions. Pakistan J. Zool.52(5):1691-1699.

DOI:https://dx.doi.org/10.17582/journal.pjz/20190704110716

Renkema J.M., B. Evans and S. Devkota. 2018. Management of Flower Thrips in Florida Strawberries with Steinernema feltiae (Rhabditida: Steinernematidae) and the Insecticide Sulfoxaflor. Florida Entomologist, 101(1):102-108. https://doi.org/10.1653/024.101.0118

Sabra, I.M., M.A. El-Nagar and M.M.I. Khewa. 2005. Efficacy of some non-chemical insecticides against Thrips tabaci lind. and its associated predators. Egypt. J. Agric. Rec. 83(2): 653-659.

Saleh, M.Z.E. 2004. Ecological studies on some onion pests and their control in Assiut Governorate. Ph. D. Thesis, Fac. Agric., Assiut Univ., Egypt, 158pp. https://search.mandumah.com/Record/534849

Sallam A.A.A., S. Aboelkassem, H.A. Fouad, M.F. Abd ElMageed. 2018. Efficiency of certain insecticides compared to selected alternatives on onion thrips (Thrips tabaci Lind.) under field conditions. Archives of Agricultural Sci. J. 1(1):58-70. DOI: 10.21608/aasj.2018.23572

Salman, A.M.A. 2000. Relative susceptibility of certain onion varieties to the infestation of the onion Thrips Tabaci (lind) and the Onion maggot, Delia alliria (Meigen) in Upper Egypt. J. Agric. Sci. Mansoura Univ.25(1):469-473. https://citeseerx.ist.psu.edu/viewdoc/download?doi=10.1.1 $.1047 .9690 \&$ rep $=$ rep $1 \&$ type $=$ pdf

SAS Institute.1989. SAS user's guide: statistics. SAS Institute, Cary, N.C.

Shah R.A. 2015. Distribution and management of Thrips tabaci (Lindeman) (Thysanoptera: Thripidea) on onion (Allium cepa) in selected districts of Khyber Pakhtunkhwa province Pakistan. PhD Thesis. Dept. of Entomol, Univ. Agric. Peshawar, pp 122. https://core.ac.uk/download/pdf/132561449.pdf

Srivastava M., J. Funderburk, S. Olson, O. Demirozer, S. Reitz. 2014. Impacts on natural enemies and competitor thrips of insecticide against the western flower thrips (Thysanoptera: Thripidae) in fruiting vegetables. Florida Entomologist. 97: 337-348. https://doi.org/10.1653/024.097.0201 
Zepa C., V. Tabara, I. Petrescu \& Palagesiu. 2011. Chemical control of Thrips tabaci attack on the crop of Calendula officinalis. Romanian Agricultural Research. 28: 243-247.

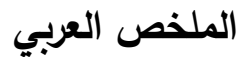 \\ كفاءة بعض المبيدات الحشرية الكيميائية والحيوية علي حشرة تربس البصل}

\section{Thrips tabaci Lindeman}

علاء مسعود حيطاوي خزيمي، محمد عبدالسلام فرج أبوزيد، عدنان عبدالفتاح السيد درويش

Red Giza وGiza المبيدات المختبرة ترتيبا تتازليا حسب كفاءتها في خفض تعداد حوريات حشرة تربس البصل كالآتي: spinetoram، lambda- ،thiamethoxam ،imidacloprid ‘pyridalyl 'malathion 'pyriproxyfen 'dinotefuran 'cyhalothrin وأخيرا Beauvaria bassiana. من ناحية أخري يمكن ترتيب هذه المبيدات ترتيبا تتازليا حسب كفاءتها في خفض تعداد الحشرات الكاملة لتربس البصل الي pyridalyl، Lambda- ،imidacloprid ،thiamethoxam 'spinetoram 'malathion 'pyriproxyfen 'dinotefuran 'cyhalothrin Beauvaria bassiana أيضا أن حشرة تربس البصل كان لها جيلين خلال فترة النمو الخضري لنباتات البصل. ووجد أن هناك ارتباط معنوي سالب بين تعداد حشرة التربس وبين الرطوبة النسبية وعلاقة ارتباط موجب بين درجات الحرارة وتعداد الحشرة.
تعتبر حشرة تربس البصل Thrips tabaci واحدة من المشاكل الرئيسية والعوامل المحدة لزراعة وانتاج البصل لردل Allium cepa L. لبعض أصناف البصل المختلفة للاصابة بحشرة تربس البصل، كذلك تم عمل مسح حقلي لكفاءة بعض المبيدات الحشرية علي حشرة تربس البصل، بالاضافة لدراسة التقلبات العددية ودراسة تاثير بعض العوامل الجوية علي تعداد الحشرة. لذلك قد تساعد هذه الدراسة في برامج المكافحة

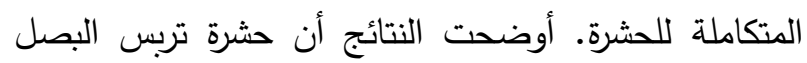
تصيب كل الاصناف المختبرة وان الصنف Red Giza كان أكثر الاصناف مقاومة للاصابة بالحشرة. من ناحية أخري كان الصنف Giza 6 هو الصنف الاكثر حساسية للاصابة ويليه الصنف Beheri ثم 20 وأخيرا الصنف Giza White هذا ولا توجد فروق معنوية بين الصنفين. هiza 